\title{
CONSTRUCTION OF SOME NEW FOUR-DIMENSIONAL MANIFOLDS
}

\author{
BY SYLVAIN E. CAPPELL AND JULIUS L. SHANESON ${ }^{1}$
}

Communicated by Edgar Brown, Jr., September 3, 1975

This note announces a new construction in the theory of 4-manifolds.

Let $\varphi: T^{3} \rightarrow T^{3}, T^{3}=S^{1} \times S^{1} \times S^{1}$, the torus of dimension three, be a diffeomorphism, with $\varphi(x)=x$, some $x \in T^{3}$. Let $A$ be a matrix for the map $\varphi$ induces on $\pi_{1} T^{3}=Z \oplus Z \oplus Z$. Assume that $\operatorname{det} A=-1$ and $\operatorname{det}\left(I-A^{2}\right)= \pm 1$, $I=$ identity matrix. It is easy to see that such a map $\varphi$ exists.

Let the manifold $M$ be obtained from $T^{3} \times[0,1]$ by identifying $(y, 0)$ with $(\varphi(y), 1)$. Let $M_{0}$ be the complement of the interior of a tubular neighborhood of the image of $\{x\} \times[0,1]$ in the quotient $M$. Clearly $\partial M_{0}$ can be identified with the boundary $S(\rho)$ of the nontrivial disk bundle $D(\rho)$ over $S^{1}$ with group $O(4)$. There is a (canonical) map $h: M_{0} \rightarrow D(\rho)$ restricting to the identity on $S(\rho)$.

Let $N$ be any connected nonorientable 4-manifold, and let $N_{0}$ be the complement of the interior of a tubular neighborhood of a circle in $N$ representing an element $\alpha \in \pi_{1} N$ that reverses orientation. Then $\partial N_{0}=S(\rho)$. Let

$$
Q_{N}=Q_{N, A}=N_{0} \cup_{S(\rho)} M_{0}
$$

and let $h_{N}=\operatorname{id}_{N_{0}} \cup h_{0}$; i.e., $Q_{N}$ is obtained from the disjoint union of $N_{0}$ and $M_{0}$ by identifying their boundaries.

Theorem. Suppose $\alpha$ has order two. Then

(i) $h_{N}$ is a simple homotopy equivalence,

(ii) $h_{N}$ is not homotopic to a diffeomorphism (or even to a PL homeomorphism).

For example, let $N$ be real projective 4-space. Then $Q_{N}$ is not diffeomorphic or even PL homeomorphic or PL s-cobordant to $N$. In fact, there are exactly two s-cobordism classes of homotopy 4-dimensional real projective spaces. In particular one has

THEOREM. There is a smooth free action of the group of order two, on a homotopy 4-sphere, that is not equivariantly diffeomorphic (or even PL homeomorphic) to a linear action on the standard 4-sphere.

AMS (MOS) subject classifications (1970). Primary 55A10, 57D60, 57D65, 57E25, 57D80; Secondary 57D55, 57A15.

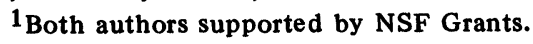




\section{REFERENCES}

S. E. Cappell, Superspinning and knot complements, Topology of Manifolds (Proc. Inst. Univ. of Georgia, Athens, Ga., 1969), Markham, Chicago, Ill., 1970, pp. 358-383. MR 43 \#2711.

S. E. Cappell and J. L. Shaneson, There exist inequivalent knots with the same complement, Ann. of Math (to appear).

COURANT INSTITUTE OF MATHEMATICAL SCIENCES, NEW YORK UNIVERSITY, NEW YORK, NEW YORK 10012 (Current address of S. E. Cappell)

Current address (J. L. Shaneson): Department of Mathematics, Rutgers University, New Brunswick, New Jersey 08903 